\title{
Synchrony of rest tremor in multiple limbs in Parkinson's disease: evidence for multiple oscillators
}

\author{
H. Ben-Pazi ${ }^{1,3}$, H. Bergman ${ }^{2,4}$, J. A. Goldberg ${ }^{2,4}$, N. Giladi ${ }^{1}$, D. Hanse ${ }^{4,5}$, \\ A. Reches ${ }^{3}$, and E. S. Simon ${ }^{1}$ \\ ${ }^{1}$ Department of Neurology, Tel-Aviv Sourasky Medical Center, Tel-Aviv, \\ ${ }^{2}$ Department of Physiology, and \\ ${ }^{3}$ Department of Neurology, Hebrew University Faculty of Medicine, Jerusalem, and \\ ${ }^{4}$ Interdisciplinary Center for Neural Computation, Hebrew University, \\ Jerusalem, Israel \\ ${ }^{5}$ Laboratorie de Neurophysique et Physiologie du Systeme Moteur, \\ EP 1848-CNRS, Paris, France
}

Accepted September 7, 2000

\begin{abstract}
Summary. Recent evidence points to involvement of central nervous system oscillators in Parkinson's disease (PD) rest tremor. It remains unknown whether one or multiple oscillators cause tremor in multiple limbs. Based on the prediction that multiple oscillators would cause low coherence even with similar average frequency, we studied 22 PD patients using accelerometers on multiple limbs. Records were digitized and spectral analysis was performed. Peak frequencies in the arms, legs, and chin were similar, indicating that biomechanical factors did not determine the frequency. Coherence between different axes of individual accelerometers and between different segments of the same limb was high. However, coherence between tremor in different limbs was low. There was no consistent pattern across patients of ipsi- vs. contralateral predominance of coherence. These data suggest that tremor in $\mathrm{PD}$ is generated by multiple oscillatory circuits, which operate on similar frequencies.
\end{abstract}

Keywords: Tremor, Parkinson's disease, oscillations, synchrony, basal ganglia, coherence.

\section{Introduction}

Rest tremor in Parkinson's disease (PD) is a common and often debilitating symptom that may be refractory to medical treatment. Recently, improved understanding of the physiology of the basal ganglia (BG) (Albin et al., 1989; Chesselet and Delfs, 1996; Wichmann and DeLong, 1996) has led to the reemergence of stereotactic neurosurgical procedures as viable and effective treatment options for PD tremor. Lesions or deep brain stimulation to either 
the ventral intermediate nucleus (VIM) of the thalamus (Benabid et al., 1991; Pollak et al., 1993), globus pallidus (Lozano et al., 1995), or subthalamic nucleus (Benabid et al., 1994; Kumar et al., 1998) can effectively alleviate tremor in contralateral limbs in PD. These developments highlight the need for better understanding of PD tremor pathophysiology, as a basis for future therapeutic innovations.

Mounting evidence implicates involvement of several CNS regions in generation and propagation of PD rest tremor. Intraoperative recordings have demonstrated "tremor cells" in the thalamus (Lenz et al., 1988), globus pallidus (Hutchison et al., 1997), and subthalamic nucleus (Hutchison et al., 1998) of PD patients undergoing stereotactic procedures for tremor relief. Additionally, data from multi-cellular recording from globus pallidus in primates made parkinsonian by administration of 1-methyl-4-phenyl-1,2,3,6tetrahydropyridine (MPTP) showed emergence of increased oscillations and synchrony of neighboring cells (Bergman et al., 1998). In other studies of PD tremor, voltage oscillations at the tremor frequency in the thalamus (Volkmann et al., 1996) and primary motor and premotor cortices (Tass et al., 1998) were found by magnetoencephalography. These observations begin to address why surgical lesions or electrical stimulation of thalamic (Benabid et al., 1991) or BG nuclei (Benabid et al., 1994; Lozano et al., 1995; Kumar et al., 1998) can effectively improve tremor in contralateral limbs in PD.

A central issue in investigation of tremorgenic mechanisms involves identification and characterization of involved nuclei or networks. It remains controversial whether one or multiple interacting tremorgenic circuits are critical. Studies of neuronal firing-tremor correlation (Hurtado et al., 1999; Levy et al., 1999) and of multi-limb tremor recording (Lauk et al., 1999; Raethjen et al., 2000) indicated that multiple oscillatory mechanisms are involved. However, Hunker and Abbs (1990), using multi-limb electromyographic (EMG) recordings showed similar tremor frequency, from which the authors inferred a single oscillatory generator. The present study involves frequency domain analysis of multi-limb accelerometric recordings to examine the relationship of PD rest tremor between different body parts. Our null hypothesis was that tremor in different body parts would be of high coherence if generated by a single oscillator. These data were previously presented in abstract form (Ben Pazi et al., 1999).

\section{Methods}

Twenty two patients at two medical centers participated in the study. Inclusion criteria was determined by a movement disorders specialist based on the diagnosis of PD according to the United Kingdom Brain Bank criteria (Hughes et al., 1992), and the existence of bilateral limb tremor. Exclusion criteria were significant dementia, concurrent neurological illness, pyramidal signs, or significant autonomic failure. All patients underwent routine neurological examinations including the United Parkinson's Disease Rating Scale (UPDRS). Patients were requested to stop levodopa the night prior to testing. The Helsinki Commission of the Health Ministry of the State of Israel approved the study, and all patients provided informed consent.

The tremor of 13 subjects was recorded by triaxial accelerometers (16 grams; Kistler, USA) and that of nine subjects was recorded by miniature monoaxial accelerometers 
(0.5 grams; Depew, USA). Accelerometers were placed variably on the index finger, hand dorsum, upper arm, foot dorsum, first toe, or chin, according to the available accelerometers and the clinical expression of tremor. Subjects were in a comfortable supine position (lying flat or reclined sitting) with hands at rest and not touching each other. Three successive 2 minutes epochs were recorded. The accelerometer's signal was amplified, hardware filtered (Butterworth, 4 poles, $1-30 \mathrm{~Hz}$ ), and digitized at a rate of $100 \mathrm{~Hz}$ (National Instruments, USA).

Digital data analysis was implemented in a custom program written in Matlab (The Mathworks, USA). The power spectra in the bandwidth of $3-18 \mathrm{~Hz}$ were estimated using Welch's method, a fast Fourier transform algorithm, and the frequency of the maximal power magnitude was determined (referred to here as "peak frequency"). The synchrony between the signals was determined by estimating the coherence function, which measures the linear correlation between them as a function of the frequency (Carter, 1987). This function results in a frequency dependent curve ranging in magnitude from $0-1$, where 1 indicates that the frequency of two signals co-vary exactly in the given frequency band. In all cases, coherence measures were evaluated for the entire data epoch to enhance resolution of the coherence function. The resolution of the power and coherence spectra was $0.2 \mathrm{~Hz}$, and the $95 \%$ confidence level for the coherence was 0.13 , based on (Carter, 1987). Maximal coherence values in the range of $3-8 \mathrm{~Hz}$ (the range of PD tremor) were considered for this study. When population summaries are given they are as "average \pm standard deviation".

In order to examine whether there was greater coherence between ipsi- or contralateral limbs in each subject, we used the following formula: (Ipsi - Contra)/(Ipsi + Contra), where Ipsi is the coherence of tremor signals from limbs on the same side of the body, and Contra is the coherence of tremor signals from homologous limbs on opposite sides of the body. We sought correlation of this tremor "ipsi/contra predominance" parameter with clinical subclass as defined by Tremor/Postural Instibility and Gait Difficulty (T/PIGD) score, and with other clinical variables such as disease duration, sex, and age. The T/PIGD score is a measure derived from the UPDRS, as an indication of the relative severity of tremor or postural instability and gait difficulties (Jankovic et al., 1990).

\section{Results}

Patient characteristics are shown in Table 1. Of note, there was a wide range of severity of PD manifestations, as evidenced by total UPDRS scores. In addition, T/PIGD scores were widely distributed. All patients were treated with levodopa/carbidopa, together with various combinations of selegeline, and dopamine agonist and anticholinergic drugs. Patients complied to various degrees with the request to withhold levodopa, but all patients had tremor at the time of examination.

Data from one patient is shown in Fig. 1, which illustrates how tremor signals of similar frequency may have either high or low coherence. Power spectra computed from long time epochs showed clear peaks indicating dominant frequency ("peak frequency") at approximately $5 \mathrm{~Hz}$. However, coherence was low between ipsilateral limbs (Fig. 1C) and high between contralateral limbs (Fig. 1F). The finding of low coherence between signals that have apparently similar frequencies may be understood in light of the fact that the power spectrum represents the average from the entire data epoch. If the frequencies of two signals change in different ways over time, then the coherence may be low despite the fact that the average frequency may be the same. Here, we see that in the same individual, relatively high 
Table 1. Clinical characteristics of the patient population. Evident in the T/PIGD is the bias of our selection for the presence of significant tremor

\begin{tabular}{lllllll}
\hline Patient & Age & Sex & $\begin{array}{c}\text { Duration } \\
\text { (years) }\end{array}$ & Side & UPDRS & T/PIGD \\
\hline MM & 60 & F & 12 & L & 52 & 2 \\
MN & 78 & M & 7 & L & 75 & 0.69 \\
DD & 67 & M & 14 & R & 36 & 0.63 \\
AS & 58 & F & 12 & L & 56 & 2.22 \\
NF & 67 & F & 5 & L & 41 & 1.67 \\
FY & 62 & M & 19 & $\mathrm{~L}$ & 55 & 4.43 \\
DZ & 67 & F & 14 & $\mathrm{R}$ & 34 & 0.89 \\
SM & 45 & M & 4 & $\mathrm{R}$ & 117 & 1.01 \\
MV & 70 & $\mathrm{M}$ & 9 & $\mathrm{R}$ & 57 & 4.44 \\
AM & 67 & $\mathrm{M}$ & 2 & $\mathrm{R}$ & 51 & 0.95 \\
AP & 84 & $\mathrm{M}$ & 15 & $\mathrm{~L}$ & 111 & 0.53 \\
BS & 73 & $\mathrm{M}$ & 5.5 & $\mathrm{R}$ & 70 & 1.49 \\
BY & 59 & $\mathrm{M}$ & 8 & $\mathrm{R}$ & 46 & 1.53 \\
GY & 51 & $\mathrm{M}$ & 17 & $\mathrm{~B}$ & 80 & 2.31 \\
CI & 62 & $\mathrm{M}$ & 5 & $\mathrm{R}$ & 93 & 0.59 \\
HM & 84 & $\mathrm{~F}$ & 12 & $\mathrm{~L}$ & 54 & 0.42 \\
LS & 68 & $\mathrm{M}$ & 6 & $\mathrm{~L}$ & 66 & 1.11 \\
CM & 53 & $\mathrm{M}$ & 9 & $\mathrm{~L}$ & 79 & 7.22 \\
NA & 72 & $\mathrm{M}$ & 1.5 & $\mathrm{R}$ & 36 & 4.44 \\
SY & 74 & $\mathrm{M}$ & 4 & $\mathrm{~L}$ & 59 & 2.41 \\
PS & 66 & $\mathrm{~F}$ & 2 & $\mathrm{~L}$ & 27 & 5.00 \\
SI & 64 & $\mathrm{M}$ & 15 & $\mathrm{R}$ & 55 & 2.08 \\
Average & 65.95 & & 9.0 & & 61.36 & 2.26 \\
\hline
\end{tabular}

UPDRS Uniform Parkinson's Disease Rating Scale; T/PIGD Tremor / postural instability and gait disorder score (computed from the UPDRS)

coherence is present in the tremor bandwidth only between contralateral hands.

On average, PD rest tremor in different limbs had similar peak frequency. This is demonstrated in Fig. 2A, where the bars represent the peak frequency for all measurements, averaged across all subjects. The surprisingly small variability in the peak frequency regardless of the mass of the limb indicates that all body parts receive similar rhythmic neuronal drive. However, it cannot be concluded based on average frequency data alone whether the tremor in different limbs was derived from the same oscillatory source. Since the peak frequency of each accelerometer signal was computed from the entire recording epoch, the similarity in peak frequency indicates only that the average frequency in the two signals was similar for that epoch. In fact, we observed that there was considerable temporal variation of individual power spectra (not shown). As such, it is possible that the two signals have different instantaneous frequencies that change over time, and therefore the temporal changes in the signals are not related. In such a case, low coherence of the two signals provides evidence for different tremorgenic sources driving the tremor in the different joints. 

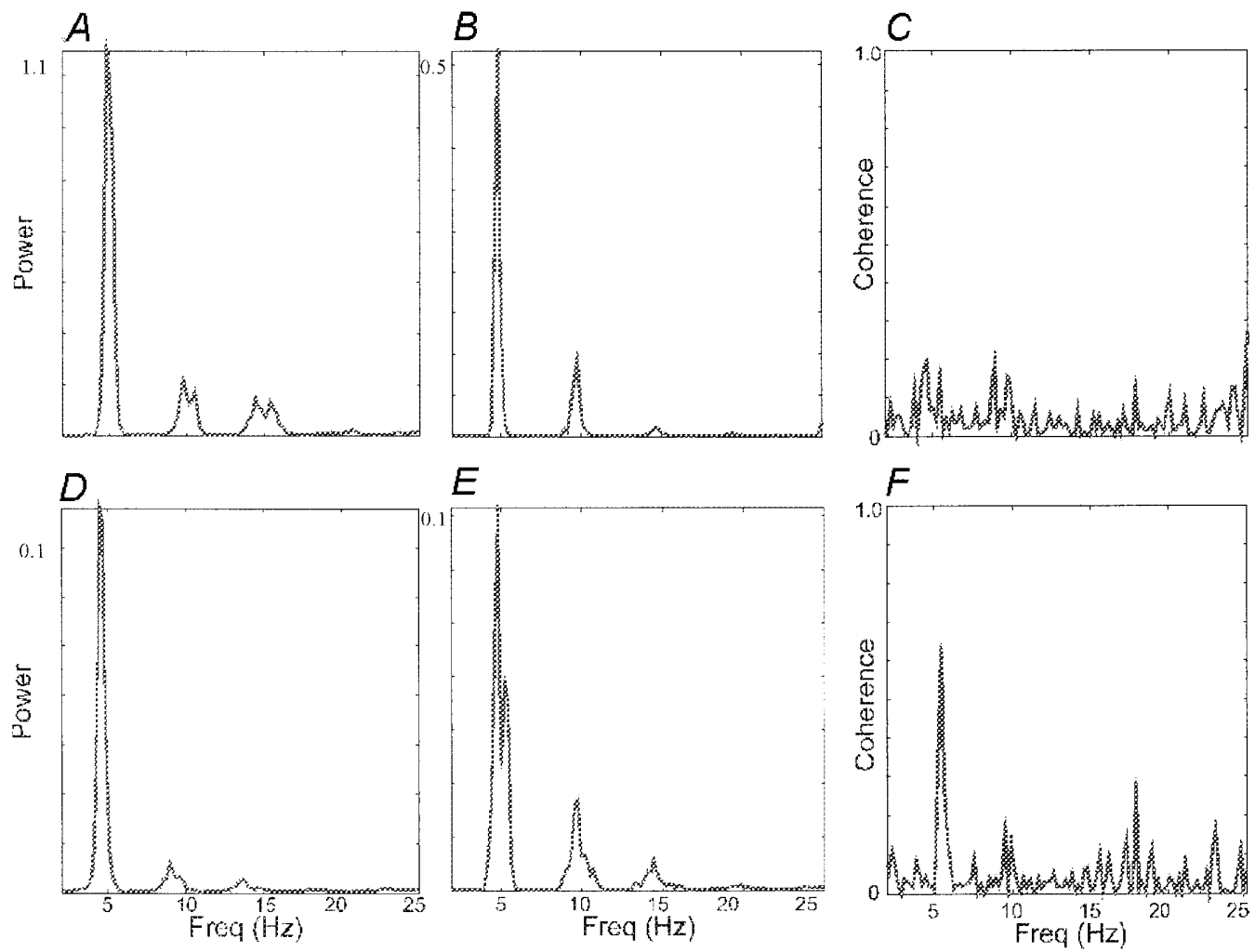

Fig. 1. Example of frequency domain data. Data from monoaxial accelerometers placed on one patient, AM, is shown after transformation of sinusoidal tremor signals to the frequency domain (see Methods). Power spectra of signals from left hand (A) and left foot (B) indicate similar peak frequency of close to $5 \mathrm{~Hz}$. However, coherence $(\mathbf{C})$ is low in the bandwidth of the tremor frequency. In contrast, during a different epoch, power spectra of signals from the left hand (D) and right hand (E) and the coherence (F) had a significant peak in the bandwidth of the tremor frequency. Units as shown; power spectra in multiples of the acceleration of gravity $(\mathrm{g})$

Several comparisons of coherence were computed to evaluate the extent of coupling between tremor signals both within and between limbs. These data, as summarized in Fig. 2B, are shown for five representative subjects. For each subject, average coherence values for comparisons between each of the axes of individual triaxial accelerometers (leftmost gray bars, "axis"), between accelerometer signals from placements on the same limb (upper arm, hand, and finger; center white bars, "segment"), and from different upper and lower limbs (rightmost black bars, "limb") were analyzed. A clear pattern emerged: while the interaxis and intersegment coherence was relatively high, the interlimb coherence was generally low. For the entire population of subjects, the interaxis coherence value was $0.72 \pm 0.19$, the intersegment coherence was $0.56 \pm 0.28$, and the interlimb coherence was $0.31 \pm 0.20$.

For six patients who had obvious chin tremor, we computed average coherence values between signals from an accelerometer placed on the chin and each of the three axes from multiple triaxial accelerometers placed on 

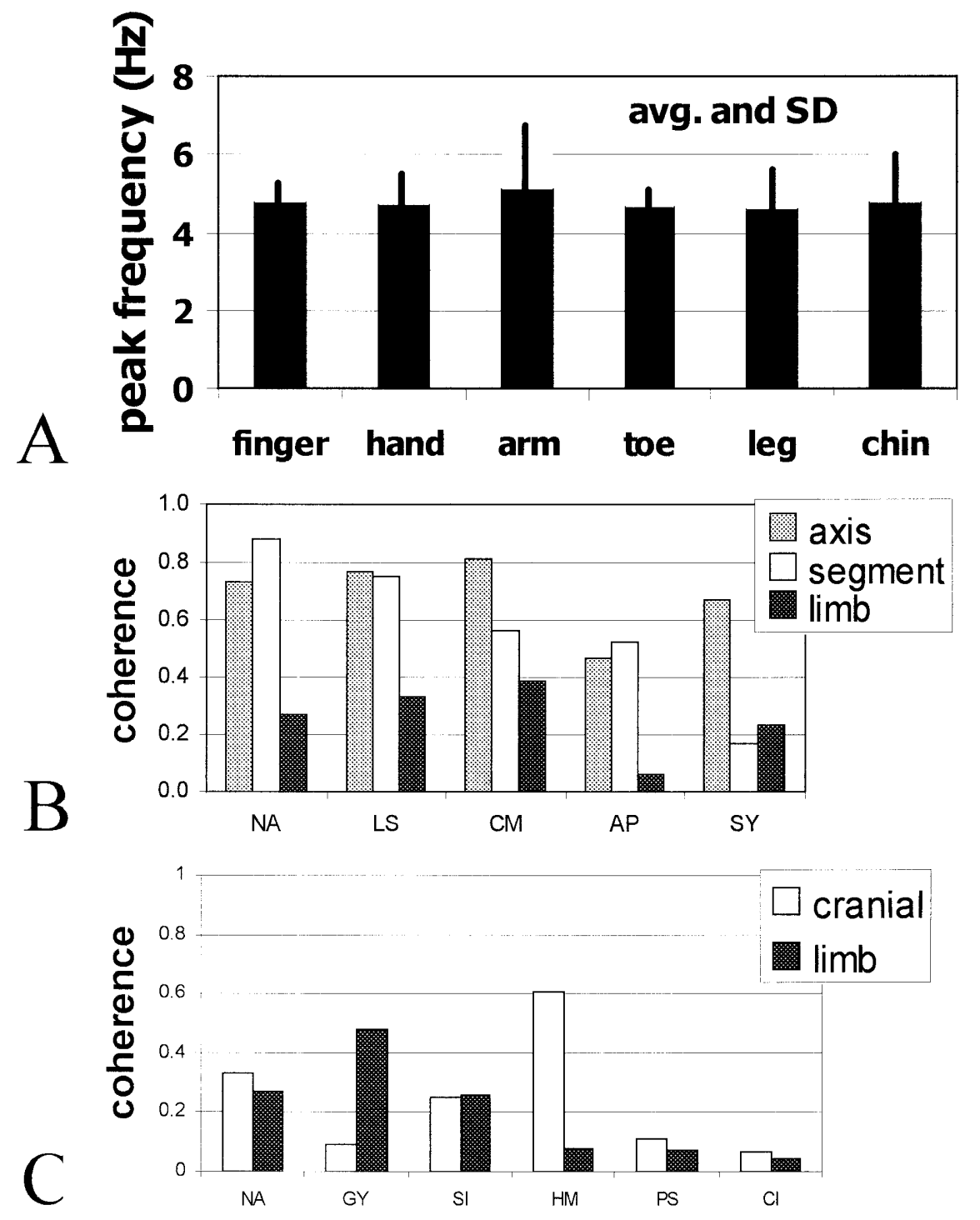

Fig. 2. Summary data: tremor frequency and coherence. A Tremor frequency in different body parts. The average and standard deviation of the peak frequency for rest tremor in different body parts is shown. Note the similarity in average frequency, despite the large difference in mass and biomechanical properties. B Intralimb and interlimb coherence. Shown is the comparison of coherence between tremor in different axes of the same accelerometer (gray; axis), between different segments of the same limb (white; segment), and between different limbs (black; limb) for a subset of patients. C Coherence between chin and limbs. Shown is the comparison of average coherence between tremor in the chin and each of the accelerometers on the limbs (white; segment), and between different limbs (black; limb) for a subset of patients. Bars represent average coherence. Coherence (ordinate) is in arbitrary units, with range $[0,1]$ (see Methods) 


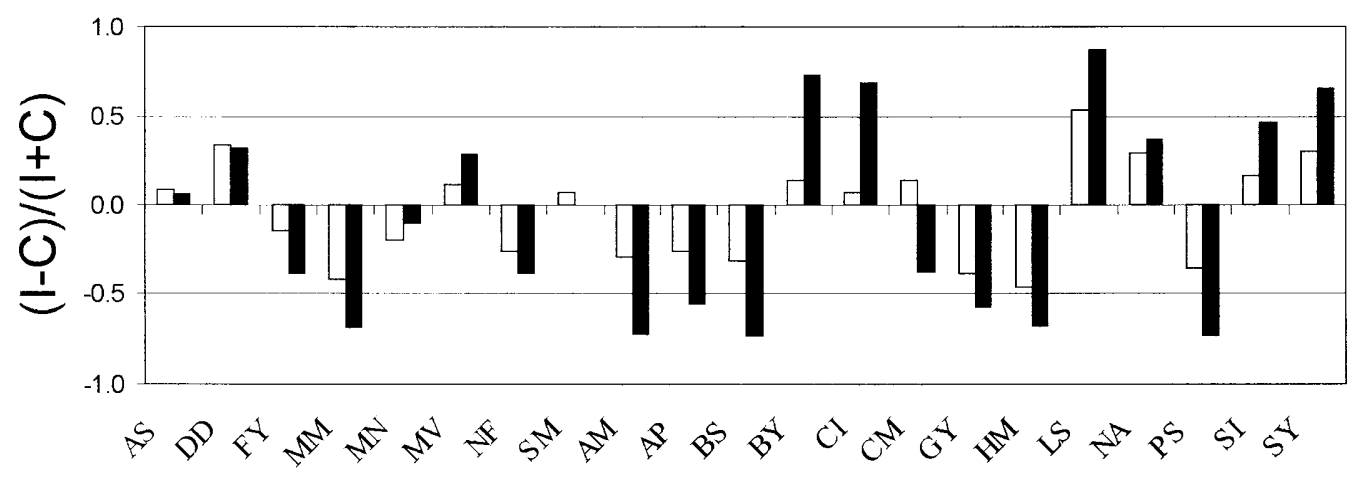

\section{Average $\mathbf{M a x}$}

Fig. 3. Comparison of coherence between ipsi- and contralateral limbs. Shown are the average (white) and maximal (black) values of the derived "ipsi/contra predominance" parameter (ordinate) for each patient (see Methods). Evident is the large variability in this parameter, both between patients and for multiple trials within each patient

the limbs. These data, summarized in Fig. 2C, demonstrate relatively low coherence, generally comparable in magnitude to interlimb coherence in the same subjects. For the entire population, chin-limb coherence was $0.22 \pm 0.23$.

The ipsi/contra predominance parameter (Ipsi - Contra)/(Ipsi + Contra), did not show any consistent pattern among the patient population (Fig. 3 ). Both the average and maximum values of this parameter are shown, indicating that there were approximately equal numbers of patients with greater coherence when comparing ipsilateral or contralateral limbs. Further, there was no significant correlation between this parameter and T/PIGD scoring, disease duration or other clinical variables (two-tailed paired t-test, $\mathrm{p}>0.2$ ).

\section{Discussion}

The present results show that, despite having similar tremor frequency, PD rest tremor in different limbs is generally of low coherence. One interpretation of these results is that tremor in different limbs is driven by different oscillators which are coupled. Since the coherence is low, we must assume that the coupling is weak and their synchrony can be easily disrupted. It should be noted that in the present study coherence was computed for long time epochs, and therefore we cannot exclude that tremor in different limbs might be more strongly synchronized for brief epochs. This issue will be a focus of future study.

An alternative interpretation of our results is that a common oscillator drives the tremor in different limbs, and that the observed low coherence might result from noise in the neural control signals downstream to the oscillator. Although we found that tremor in different limbs can display significant coherence, the magnitude was generally small. A further argument against this interpretation is that the coherence between axes of the same 
accelerometer and within limbs was systematically higher that between limbs. There should be no reason why desynchronizing noise would preferentially affect motor circuits leading to different limbs, although we cannot exclude this factor. Rather, we favor the hypothesis that different oscillators that are weakly coupled drive tremor in different limbs.

Our findings are consistent with several recent reports suggesting multiple oscillators in PD tremor (Hurtado et al., 1999; Lauk et al., 1999; Raethjen et al., 2000), physiological tremor (Lauk et al., 1999), and essential tremor (Simon and Giladi, 1999; Lauk et al., 1999). However, Hunker and Abbs (1990) suggested a single oscillator. In fact, our finding of similar average frequency in different limbs is consistent with that of Hunker and Abbs (1990). By calculating the coherence between tremor signals, a step not performed by Hunker and Abbs, we were able to infer multiple tremorgenic oscillatory circuits.

It is noteworthy that there was marked interpatient variability in the coherence measurements. Nonetheless, within each patient there was a consistent finding of low interlimb coherence relative to the interaxis and intersegment coherence. The relatively high interaxis and intersegment coherence may be accounted for either by higher mechanical coupling or tighter coupling of CNS oscillators. The likelihood of a significant degree of mechanical coupling and the high sensitivity of coherence values to such coupling (Tass et al., 1998) strengthens our conclusions, as it may be inferred that the coherence measure would be even lower in the absence of mechanical coupling.

As a step towards understanding the somatotopic organization of tremorgenic oscillators in PD, we sought a measure of the degree of unilaterality of the tremor coherence. Since most motor circuits are mainly related to one side of the body and PD signs are often asymmetric, it may be expected that coherence of tremor would be greater between ipsilateral limbs than in contralateral limbs. However, we found no pattern of the "ipsi/contra predominance" parameter across our patient population. Regarding clinicalelectrophysiological correlation, we postulated that the degree of unilaterality might correlate with the subclass of PD - either those patients exhibiting a tremor-predominant syndrome or patients with a greater tendency towards bilateral symptoms of postural instability and gait disability. We predicated that the tremor-predominant patients would have greater unilaterality, as reflected by the "ipsi/contra predominance" parameter. However, we found no significant correlation between these two measures. We are aware that the small numbers of patients in this study precludes definitive statements regarding patient subclass characteristics.

In conclusion, the present results demonstrate low interlimb coherence in PD rest tremor, and thereby provide evidence for multiple oscillators in tremorgenesis. This provides orientation for experimental design and data interpretation for studies aimed at identifying and characterizing CNS networks involved in tremor generation, employing extracellular recording and neuroimaging techniques. We believe in the importance of further characterization of the dynamics of putative neural oscillators that 
may be involved in voluntary and involuntary movements. Studies such as this may lead to future innovations in physiologically based treatments for PD.

\section{Acknowledgments}

The authors are grateful for the support and guidance of Prof. A. Korczyn. This work was supported in part by Israeli Academy of Science, AFIRST, and the US-Israel Bi-national Science Foundation (HB).

\section{References}

Albin RL, Young AB, Penney JB (1989) The functional anatomy of basal ganglia disorders. Trends Neurosci 12: 366-375

Ben Pazi H, Giladi N, Korczyn AD, Reches A, Hansel D, Goldberg J, Bergman H, Simon ES (1999) Low interlimb coherence in Parkinson's disease rest tremor [abstract]. Soc Neurosci Abstr 25: 375

Benabid A, Pollak P, Gervason C, Hoffmann D, Gao D, Hommel M, Perret J, De Rougemont J (1991) Long-term suppression of tremor by chronic stimulation of the ventral intermediate thalamic nucleus. Lancet 337: 403-406

Benabid AL, Pollak P, Gross C, Hoffmann D, Benazzouz A, Gao DM, Laurent A, Gentil M, Perret J (1994) Acute and long-term effects of subthalamic nucleus stimulation in Parkinson's disease. Stereotact Funct Neurosurg 62: 76-84

Bergman H, Feingold A, Nini A, Raz A, Slovin H, Abeles M, Vaadia E (1998) Physiological aspects of information processing in the basal ganglia of normal and parkinsonian primates. Trends Neurosci 21: 32-38

Carter GC (1987) Coherence and time delay estimation. Proc IEEE 75: 236-255

Chesselet MF, Delfs JM (1996) Basal ganglia and movement disorders: an update. Trends Neurosci 19: 417-422

Hughes A, Daniel S, Kilford L, Lees A (1992) Accuracy of clinical diagnosis of idiopathic Parkinson's disease: a clinico-pathological study of 100 cases. J Neurol Neurosurg Psychiatry 55: 181-184

Hunker C, Abbs J (1990) Uniform frequency of parkinsonian resting tremor in the lips, jaw, tongue, and index finger. Mov Disord 5: 71-77

Hurtado JM, Gray CM, Tamas LB, Sigvardt KA (1999) Dynamics of tremor-related oscillations in the human globus pallidus: a single case study. Proc Natl Acad Sci USA 96: 1674-1679

Hutchison WD, Lozano AM, Tasker RR, Lang AE, Dostrovsky JO (1997) Identification and characterization of neurons with tremor-frequency activity in human globus pallidus. Exp Brain Res 113: 557-563

Hutchison WD, Allan RJ, Opitz H, Levy R, Dostrovsky JO, Lang AE, Lozano AM (1998) Neurophysiological identification of the subthalamic nucleus in surgery for Parkinson's disease. Ann Neurol 44: 622-628

Jankovic J, McDermott M, Carter J, Gauthier S, Goetz C, Golbe L, Huber S, Koller W, Olanow C, Shoulson I, et al. (1990) Variable expression of Parkinson's disease: a base-line analysis of the DATATOP cohort. The Parkinson Study Group. Neurology 40: $1529-1534$

Kumar R, Lozano AM, Kim YJ, Hutchison WD, Sime E, Halket E, Lang AE (1998) Double-blind evaluation of subthalamic nucleus deep brain stimulation in advanced Parkinson's disease. Neurology 51: 850-855

Lauk M, Koster B, Timmer J, Gusclauber B, Deuschl G, Lucking CH (1999) Side-to-side correlation of muscle activity in physiological and pathological human tremors. Clin Neurophysiol 110: 1774-1783

Lenz FA, Tasker RR, Kwan H, Schneider S, Kwong R, Murayama Y, Dostrovsky JO, Murphy J (1988) Single unit analysis of the human ventral thalamic nuclear group: 
correlation of thalamic "tremor cells" with the $3-6 \mathrm{~Hz}$ component of parkinsonian tremor. J Neurosci 8: 754-764

Levy R, Davis KD, Hutchison WD, Pahapill PA, Lozano AM, Tasker RR, Dostrovsky JO (1999) Simultaneously recorded neuron pairs in the motor thalamus of patients with Parkinson's disease and essential tremor [Abstract]. Soc Neurosci Abstr 25: 14081408

Lozano AM, Lang AE, Galvez Jimenez N, Miyasaki J, Duff J, Hutchinson WD, Dostrovsky JO (1995) Effect of GPi pallidotomy on motor function in Parkinson's disease. Lancet 346: 1383-1387

Pollak P, Benabid AL, Gervason CL, Hoffmann D, Seigneuret E, Perret J (1993) Longterm effects of chronic stimulation of the ventral intermediate thalamic nucleus in different types of tremor. Adv Neurol 60: 408-413

Raethjen J, Lindemann M, Schmaljohann H, Wenzelburger R, Pfister G, Deuschl G (2000) Multiple oscillators are causing parkinsonian and essential tremor. Mov Disord 15: 84-94

Simon ES, Giladi N (1999) Essential tremor is essentially different from physiological tremor: a study of position-specific intra-limb frequency and phase relationships [Abstract]. Neurology 52: A518

Tass P, Volkmann J, Weule J, Schnitzler A, Witte OW, Freund H-J (1998) Corticomuscular and corticostriatal synchronization in parkinsonian rest tremor as revealed by magnetoencephalography [Abstract]. Mov Disord 13 [Suppl 12]: 268-268

Volkmann J, Joliot M, Mogilner A, Ioannides A, Lado F, Fazzini E, Ribary U, Llinas R (1996) Central motor loop oscillations in parkinsonian resting tremor revealed by magnetoencephelography. Neurology 46: 1359-1370

Wichmann T, Delong MR (1996) Functional and pathophysiological models of the basal ganglia. Curr Opin Neurobiol 6: 751-758

Authors' address: E. S. Simon, M.D., Laboratory of Motor Physiology, Department of Neurology, Tel-Aviv Sourasky Medical Center, 6 Weizmann Street, Tel-Aviv, 64239 Israel, e-mail: ely_tasmc@yahoo.com 